\title{
The backlash against bioequivalence and the interchangeability of brand-name and generic drugs
}

\author{
Aaron S. Kesselheim MD JD
}

See related research article by Tsadok and colleagues at www.cmaj.ca/lookup/doi/10.1503/cmaj.101800 and synopsis on page 1371 ; see also practice article by Mackenzie and colleagues on page 1393 and at www.cmaj.ca/lookup/doi/10.1503/cmaj.100351.

Competing interests: None declared.

This article was solicited and has not been peer reviewed.

Correspondence to: Dr. Kesselheim, akesselheim@partners.org

CMAJ 2011. DOI:10.1503 /cmaj.110808
1 s recently as the 1960 s, it was illegal in many jurisdictions for a pharmacist to substitute a generic drug for the brandname version a physician had prescribed. Since that time, manufacturers of generic drugs have grown from small, shoestring operations to include some of the world's largest corporations, and laws mandating the interchangeability of generic and brand-name medications have become the norm. Now, generic drugs account for three-quarters or more of all prescriptions filled, but only about $20 \%$ of spending on pharmaceuticals. Generic drugs have well-described health benefits, as patients prescribed lower-cost generic drugs are more likely to adhere to their treatment regimens. ${ }^{1}$

Despite the steady growth of the market for generic drugs, skepticism regarding their use has persisted. Accounts expressing uncertainty usually emphasize the possibility that differences might exist between brand-name and generic drugs that could lead to idiosyncratic adverse effects or systematic differences in drug efficacy. The basis for such concerns is the concept of "bioequivalence." Generic drugs are approved by national regulatory bodies, such as Health Canada and the US Food and Drug Administration (FDA), based on proof of similarity to their brand-name counterparts in pharmacodynamic studies - similarities in parameters such as maximum serum concentration and time to maximum concentration. Most regulatory bodies require bioequivalence parameters to fall within a $25 \%$ window, meaning that one generic version could conceivably have $80 \%$ bio- equivalence to the reference product, whereas another could have $125 \%$ bioequivalence.

Is bioequivalence sufficient from a clinical point of view? In the medical literature, case studies have reported instances in which patients have had adverse outcomes related to the use of generic in place of brand-name formulations, ${ }^{2}$ leading many editorialists to warn against substitution. ${ }^{3}$ Most of these physicians express particular concern over drugs with a narrow therapeutic index, such as antiepileptic drugs, immunosuppressive agents, anticoagulant agents and antiarrhythmic products such as digoxin and amiodarone. For example, the American Academy of Neurology declared opposition to "generic substitution of anticonvulsant drugs for the treatment of epilepsy without the attending physician's approval." 4 The popular media have picked up on such views, with journalists highlighting the experiences of patients who found "stark differences among drugs the FDA has deemed equivalent"s and concluding that generic drugs are a "bad bargain." 5 These opinions are bolstered by marketing campaigns organized by the manufacturers of brand-name drugs that cast doubt on the effectiveness of generic drugs. ${ }^{6}$

However, even among drugs with narrow therapeutic indices, little rigorous evidence supports the backlash against bioequivalence. In a recent review of bioequivalence studies conducted between 1996 and 2007, the FDA found that the average difference in pharmacodynamics_between generic and brand-name products was about $4 \%$; in nearly $98 \%$ of the studies reviewed, the properties of generic products differed from those of the brand-name product by less than $10 \% .^{7}$ Although case reports of differences in outcomes among particular patients cannot draw valid causal inferences about associations, the few randomized controlled trials of differences between brand-name and generic drugs that exist have failed to show important differences in clinical outcomes. ${ }^{3}$ However, these studies may be underpowered to detect true clinical differences.

Some skeptics of bioequivalence have pointed to observational studies involving patients taking antiepileptic agents. These studies show a high rate

All editorial matter in CMAJ represents the opinions of the authors and not necessarily those of the Canadian Medical Association. 
of "switchback" or an increased use of health care services by patients who switched from brandname to generic formulations. ${ }^{8}$ In contrast, a wellcontrolled study of antiepileptic drugs that addressed confounders (such as the baseline status of disease among participants) found no evidence that generic substitution was associated with exacerbations of disease. ${ }^{9}$

In this issue of $C M A J$, Tsadok and colleagues ${ }^{10}$ report the results of a retrospective cohort study involving patients using amiodarone - a drug with a narrow therapeutic index for which differences in pharmacodynamics between brand-name and generic formulations are presumed but rarely rigorously studied. Their main finding relates to rates of drug-related thyroid dysfunction, and they found no differences between people who used the brandname formulation of amiodarone and people who used generic versions. Their study is novel in its focus on safety, supporting the clinical relevance of bioequivalence in yet another class of drugs.

Despite the lack of high-quality data supporting real clinical differences between brand-name and generic drugs, some legislators in the US and Europe have already passed limits on generic substitution for certain classes of drugs, such as antiepileptic and immunosuppressive agents, and more seem prepared to do the same. ${ }^{11}$ Regulatory bodies such as the FDA are under political pressure to tighten their bioequivalence standards (Health Canada has already set heightened requirements for a handful of drugs with narrow therapeutic indices) ${ }^{12}$ although there is no suggestion that such a move will help allay patients' fears or lead medical societies to change their position statements. Debates over definitions of adequate bioequivalence have also slowed the attempts of numerous countries to approve follow-on products that can be substituted for brand-name biologic drugs, which account for an increasing proportion of spending on prescription drugs.

Mistrust of bioequivalence has real clinical implications. With negative reports about the interchangeability of brand-name and generic drugs in the medical literature, popular media, and medical professional societies, patient uncertainty about generic drugs remains strong,,$^{13}$ and some physicians and patients may try to avoid generic substitution. However, marking "dispense as written" on a prescription form can lead to decreased rates of filling prescriptions. ${ }^{14}$ Notably, Tsadok and colleagues found that people using the brand-name version of amiodarone (who had a slightly higher median family income) were more likely to stop taking the drug ${ }_{10}^{10}$ suggesting that generic prescriptions might promote adherence to their treatment.

Decades of experience and numerous clinical studies suggest that patients and physicians can be confident in the bioequivalence of brand-name and generic drugs approved by Health Canada, the FDA or other similar regulatory authorities. In the rare circumstances where there is concern over interchangeability, such as for high-risk patients, it may be reasonable for physicians to take extra precautions, such as additional monitoring, when substitution occurs. In addition, Tsadok and colleagues help show how policymakers and physicians should work together to address any remaining questions with well-controlled trials that have clear and clinically relevant end points, rather than by voicing unfounded skepticism or building legislative barriers to substitution.

\section{References}

1. Shrank WH, Asch SM, Joseph GJ, et al. The implications of choice: prescribing generic or preferred pharmaceuticals improves medication adherence for chronic conditions. Arch Int Med 2006;166:332-7.

2. Reiffel JA, Kowey PR. Generic antiarrhythmics are not therapeutically equivalent for the treatment of tachyarrhythmias. Am J Cardiol 2000;85:1151-3

3. Kesselheim AS, Misono AS, Lee JL, et al. Clinical equivalence of generic and brand-name drugs used in cardiovascular disease: a systematic review and meta-analysis. JAMA 2008;300:2514-26.

4. Liow K, Barkley GL, Pollard JD, et al. Position statement on the coverage of anticonvulsant drugs for the treatment of epilepsy. $\mathrm{Neu}$ rology 2007;68:1249-50.

5. Eban K. Bad bargain? Self 2009 June. Available: www.self.com /health/2009/06/dangers-of-generic-drugs?currentPage=1 (accessed 2011 May 18).

6. Huckman M. Pfizer's Lipitor vs. generics: heart of the matter for me. CNBC. 2007 Oct. 29. Available: www.cnbc.com/id/21528009 (accessed 2011 May 18).

7. Davit BM, Nwakama PE, Buehler GJ, et al. Comparing generic and innovator drugs: a review of 12 years of bioequivalence data from the United States Food and Drug Administration. Ann Pharmacother 2009;43:1583-97.

8. Duh MS, Paradis PE, Latrémouille-Viau D, et al. The risks and costs of multiple-generic substitution of topiramate. Neurology 2009;72:2122-9.

9. Devine ST, Weisbart E, Barron J, et al. Acute epilepsy exacerbations in patients switched between A-rated anti-epileptic drugs. Curr Med Res Opin 2010;26:455-63.

10. Tsadok MA, Jackevicius CA, Rahme E. Amiodarone-induced thyroid dysfunction: brand-name versus generic formulations. CMAJ 2011;183:E817-23.

11. Condition-specific drug substitution legislation: epilepsy. Denver (CO) and Wshington (DC): National Conference of State Legislatures; 2011. Available: www.ncsl.org/default.aspx?tabid $=14424$ (accessed 2011 May 18).

12. Hamburg MA. Information regarding anti-epileptic drugs: U.S. Food and Drug Administration in response to requests in Senate Report No. 111-39 and House Agricultural Committee Report No. 111-279. Available: www.hpm.com/pdf/FDA\%20Anti $\%$ 20Epileptic\%20Drug\%20Report\%20to\%20Congress\%20-\%20 2011.pdf (accessed 2011 May 18)

13. PrescriptionSolutions. Survey: nearly one-third don't know or believe generics have same active ingredients and effectiveness as brand-name drugs. 2009 June 22. Available: www .prescriptionsolutions.com/RxSolWeb/mvc/staticContent.do?page URL=/rxsol/chp/rxsolutions_com/consumer/about_us/press_release /20090625_generics.html (accessed 2011 June 22).

14. Shrank WH, Liberman JN, Fischer MA, et al. The consequences of requesting "dispense as written." Am J Med 2011;124:309-17.

Affiliations: Aaron Kesselheim is with the Division of Pharmacoepidemiology and Pharmacoeconomics, Department of Medicine, Brigham and Women's Hospital and Harvard Medical School, Boston, Mass.

Funding: Dr. Kesselheim is supported by a career development award from the Agency for Healthcare Research and Quality (K08HS18465-01), and a Robert Wood Johnson Foundation Investigator Award in Health Policy Research. 